\title{
Microscopic and mesoscopic simulation of entropic micelles
}

\author{
Peter G. Bolhuis ', Daan Frenkel* \\ FOM Institute for Atomic and Molecular Physics, Kruislaan 407, 1098 SJ Amsterdam, The Netherlands
}

\begin{abstract}
We present simulations of a purely athermal model for surfactant solutions. Using semi-grandensemble simulations, we determine the (excess) free energy of micelles as a function of the cluster size. From this information, we compute the critical micelle concentration. Subsequently, we use the information of the dilute micellar system as input for a coarse-grained polydisperse hard-sphere simulation. The aim of this simulation is to study the effect of inter-micellar interactions on the size distribution. We find that, to a first approximation, the effect of these interactions can be accounted for by a mean-field theory - the size distribution of interacting micelles is equal to that of non-interacting micelles at a lower chemical potential. The techniques used in this paper can be used to simplify the numerical study of realistic models of surfactant solutions.
\end{abstract}

\section{Introduction}

There are two ways to use computers in the study of many-body systems. The first approach, computer simulation, aims to reproduce or predict the properties of real materials. The role of the second approach, performing computer experiments to test theoretical concepts, is less obvious. In fact, whereas the potential use of computer simulation was quickly and widely appreciated [1], it took several decades for the initially uneasy relation between simulation and theory to transform into an almost perfect symbiosis. Ben Widom played an important role in this transformation through the introduction of a variety of simple models for complex systems. Examples are the Widom-Rowlinson model for binary mixtures [2] and lattice models for surfactant solutions $[3,4]$. These models and their offspring are simple but far from trivial, and a combination of theoretical analysis and numerical simulation is needed to explore their behavior.

\footnotetext{
* Corresponding author.

' Present address: Department of Chemistry, University of California, Berkeley, CA 94720, USA.
} 
In the present paper, we consider a model for surfactant solutions. We are interested in micelle formation. Our first aim is to determine the free energy of surfactant $n$-mers in solution, with $n$ ranging from one to the maximum number that is mechanically stable. We do this by performing semi-grand canonical Monte Carlo simulations [5]. For dilute surfactant solutions, i.e. when interactions between different surfactant $n$-mers can be ignored, knowledge of this free energy allows us to predict the cluster-size distribution as a function of the surfactant fugacity. It also allows us to compute the critical micelle concentration (CMC). At higher surfactant concentration interactions between micelles become important and change the size distribution. We use our numerical information about the radius and free-energy of isolated micelles to perform a "coarse-grained" grand-canonical Monte Carlo simulation to study the effect of micelle interactions on the cluster-size distribution.

The work described in the present paper differs in several respects from earlier numerical studies of micelle formation [6-9]. Partly, the differences are technical (but important): the use of semi-grand and grand-canonical simulations greatly speeds up the equilibration of the system and allows us to determine the micelle free energy with high accuracy. To our knowledge, earlier attempts to measure the size distribution of micelles, follow the opposite route: the total surfactant concentration is fixed and the free-energy of the surfactant $n$-mers is deduced from the size distribution [6-9]. Such an approach makes it difficult to separate inter- and intra-micellar contributions to the free energy, and a time-consuming extrapolation procedure to low surfactant concentrations [7] is required to determine the free energy of isolated micelles. For off-lattice models $[6,8]$ the approach has been to simply assume that inter-micellar interactions can be ignored at low (but not very low) surfactant concentrations. Below, we show that it is relatively simple to determine the micelle size-distribution from a semi-grand canonical simulation.

The model that we study shares some features with the Widom-Rowlinson model [2]. The solvent is modeled by hard spheres with diameter $D$. The surfactants are linear molecules with a head-group that is identical to the solvent particles. In addition, the surfactants have a sphero-cylindrical tail that has the same diameter as the head group. The length of the cylindrical part is denoted by $L$. For $L=0$, the surfactants would be dumbbells. To make this molecule behave like a surfactant, we assume that the spherocylindrical sections of surfactant molecules do not interact with each other. Clearly, this property makes it very advantageous for the surfactant molecules to aggregate. As we were interested in the aggregation of pure surfactants, we did not introduce a second solvent phase ("oil"). We stress that this model is not unique. Hence, we have to justify our choice for the different ingredients. First of all, we choose hard spheres to model the solvent: we do this because hard spheres are a good model for simple liquids. Secondly, we choose hard spheres for the head-groups of the surfactants. This we do because repulsion between head-groups is an important feature in real surfactants. Finally, we ignore all interactions between the surfactants tails. This is clearly an oversimplification. However, in certain limits it is correct - for instance, if the surfactants consist of a spherical head-group and an infinitely thin, rigid tail. We stress that, in the 
present model, surfactants tend to aggregate for entropic rather than energetic reasons. In fact, like the hard-sphere model and the Widom-Rowlinson model, the present model is completely athermal.

\section{Semi-grand ensemble simulations}

Most published simulations of micelle formation in surfactant solutions were performed in the constant-NVT ensemble, with the total surfactant concentration fixed. In those studies, the free energy of micelles containing $n$ surfactant molecules, is deduced from the probability to observe such an $n$-particle cluster. This analysis is not simple because it is necessary to extrapolate the probability distribution to infinite dilution. Yet, even at fairly low surfactant concentrations, several micelles may form and intermicellar interactions have a noticeable effect on the free energy. Hence, in order to measure the free energy of formation of individual micelles in the canonical ensemble one needs to simulate at extremely low surfactant concentration. This requires very large system sizes to obtain reasonable statistics. The alternative is to study a relatively small system with a single micelle in equilibrium with a reservoir of an (ideal) solution at very low surfactant concentration. In principle, we could have performed grand-canonical ensemble simulations to maintain chemical equilibrium between the single micelle and the surfactant reservoir. In such a grand canonical simulation the surfactant chemical potential of the micellar system is imposed by inserting and extracting surfactant molecules. However, a naive implementation of the grand-canonical Monte Carlo method is also inefficient because this scheme involves attempts to insert a (large) surfactant molecule at a random position in the dense liquid - the probability that such a trial move is accepted is very low. The acceptance probability can be greatly improved by working in the semi-grand ensemble [5] where instead of trying to insert a surfactant molecule, attempts are made to change a solvent molecule into a surfactant or vice versa. This corresponds to imposing the chemical potential difference between a surfactant and a solvent molecule instead of the absolute chemical potential of the surfactants. The acceptance rule for randomly selecting a particle and changing its species from $a$ to $b$ is simply:

$$
P_{\mathrm{acc}}=\min \left[1, \exp \left(-\beta \Delta U_{a \rightarrow b}+\beta \Delta \mu_{a \rightarrow b}\right)\right],
$$

where $\beta=1 / k T$ is the inverse temperature, $\Delta U_{a \rightarrow b}$ is the energy difference involved in changing the species of the particle and $\Delta \mu_{a \rightarrow b}$ is the imposed chemical potential difference between the two species. As we wish to study a single micelle in a large reservoir, the semi-grand simulations are best carried out at constant pressure [10]. In what follows, we shall use $k T$ as our unit of energy and $D$, the diameter of the surfactant head-group, as our unit of length. Hence, instead of $\mu / k T$ we write $\mu$, instead of $P D^{3} / k T$, we write $P$ and instead of $\rho D^{3}$, we write $\rho$. 


\section{Results}

We performed several isobaric semi-grand simulations on a 504 particle system of a single micelle in a hard sphere solvent with an imposed chemical potential difference between surfactant and solvent of $\Delta \mu=4.0,4.2,4.4,4.6,4.8,5.0,5.2$ and 5.4. The length of the cylindrical surfactant tail was taken to be $L=2$. The pressure was fixed at $P=8$, which corresponds to a hard sphere volume fraction of $\phi \approx 0.35$. About $4 \times 10^{5}$ Monte Carlo cycles were used for data collection. On average, during a cycle, a translational (and rotational) move as well as an identity change was attempted for every particle. In addition, volume changes were attempted on average once every cycle. The acceptance ratio for volume changes was kept at $40-50 \%$. For translational and rotational moves, the acceptance probability was kept between $20 \%$ and $30 \%$. At the imposed pressure of $P=8$, the acceptance probability of an identity swap was around 0.001 and appeared almost independent of the micelle size. At this stage of the simulation, we did not allow the number of surfactant molecules in a cluster to become smaller than 10 or larger than 70 , to avoid micelle "evaporation" on the one hand and unlimited growth of the micelle on the other. The simulations showed that when the size of a micelle is larger than approximately 70 it becomes unstable and splits into two separate clusters. Those clusters will move apart and grow independently further until they become unstable again. If the chemical potential difference of the surfactant is high enough this process will continue until a large fraction of the total volume of the system is filled with surfactant. On the other hand, if a micelle becomes smaller than 10 it rapidly shrinks and disappears, because changing a solvent into a surfactant becomes highly improbable for small clusters, while the reverse move is always feasible. Fig. 1 shows a typical snapshot of a micellar configuration obtained in the semi-grand-canonical simulations.

The free energy of a micelle of a certain size $n$ is related to the size probability distribution $P(n)$ by

$$
F_{n}=-\log P(n)+n \Delta \mu+\text { const . }
$$

The free energy of a micelle that we are interested in is actually the excess free energy of the micelle with respect to the free energy of unclustered surfactants. To compute this excess free energy, we need to sample micelles of sizes smaller than 10 . In order to cover the micellar size range from 1 to 15 we used an umbrella sampling technique $[11,10]$. In these simulations, we bias the sampling in such a way that we get good statistics on number of micelles in this size range. To get good statistics throughout this range, it is best if every micelle size in this range occurs with about the same probability. To achieve this, we chose the biasing potential in the umbrella to be approximately equal to $-F_{n}$. Of course, we do not know $F_{n}$ a priori. Therefore, the umbrella simulation was performed in an iterative way. First, a simulation was performed without using a biasing potential, at an imposed chemical potential difference of $\Delta \mu=4.8$. Subsequently, the free energy $F_{n}$ was calculated from the size distribution using equation 2. The negative of this free energy function was used as a biasing potential in the next iteration of the umbrella sampling. The new free energy $F_{n}$ was 


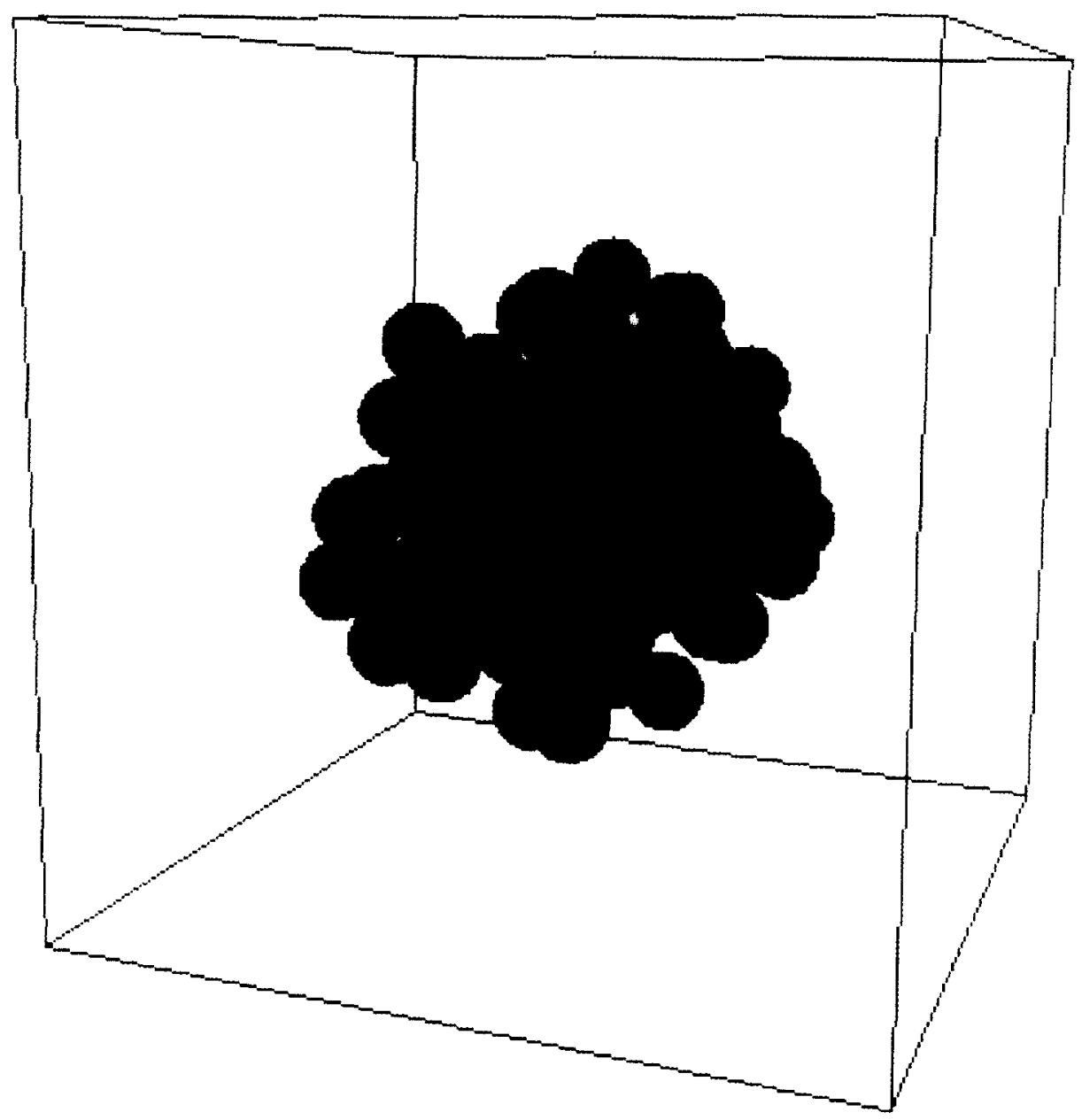

Fig. 1. Snapshot of a typical micelle generated during the semi-grand canonical simulations. This particular micelle contains 53 surfactants. In the figure, the (hard-sphere) solvent is not shown. The spheres on the outside denote the micelle head-groups. The surfactant tails inter-penetrate and their sphero-cylindrical shape is therefore not easily recognized. Clearly, the resulting micelle is fairly spherical. Note that the head groups on the surface of the micelle are not very densely packed.

calculated from the size distribution added with the biasing potential. This procedure was iterated until the distribution was almost flat.

From the size distributions at different chemical potential differences we can deduce the micellar free energy as a function of cluster-size, using Eq. (2). In this way, we obtain a number of partial free-energy curves that will, in general, differ by additive constants that remain to be determined. To combine the simulation results for different values of the imposed chemical potential, we fitted all data-sets to the same sixth order polynomial. The unknown additive constants are treated as additional fitting parameters. The resulting free energy curve is plotted in Fig. 2 for $\Delta \mu=4.8$. Clearly, there is a 


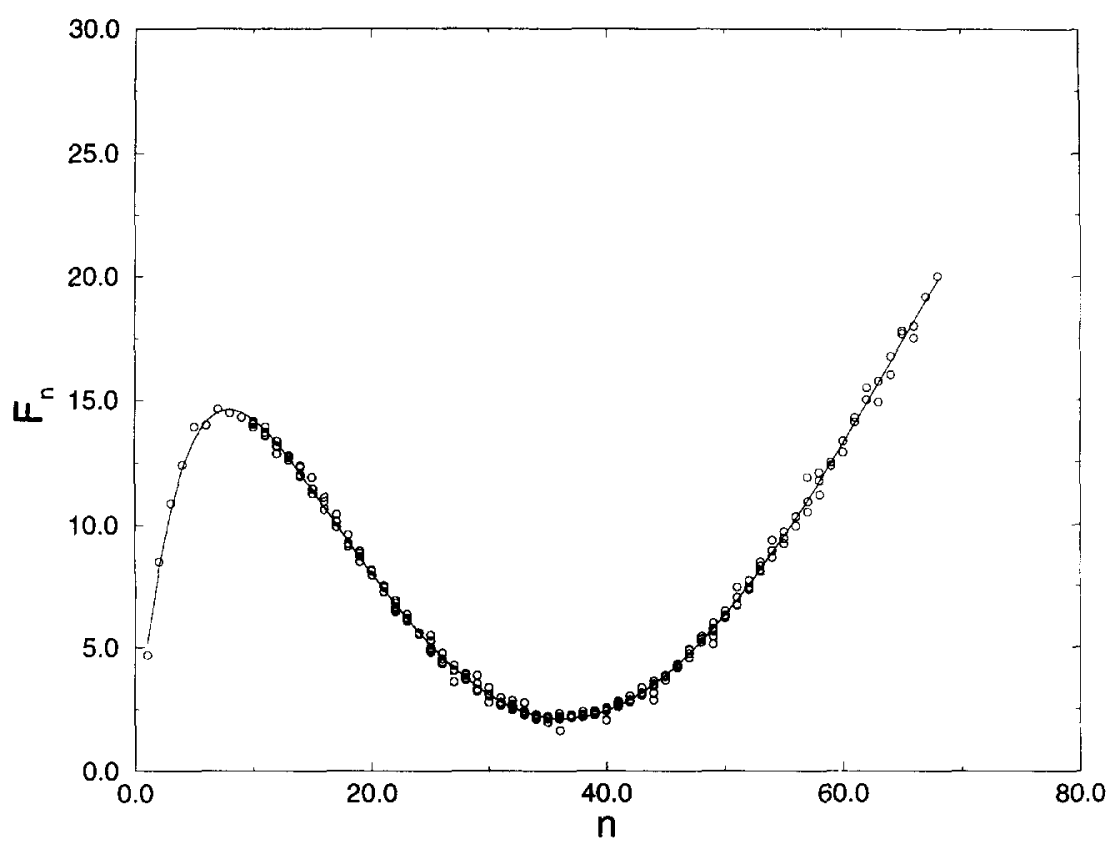

Fig. 2. Free energy of a single micelle as a function of the size (number of surfactant molecules in the micelle) for a chemical potential difference of $\Delta \mu=4.8$ and at $P=8$.

large barrier between the single surfactant and the formation of a single micelle. In most theories of micelle formation, the quantity of central interest is the (excess) free energy per surfactant molecule, as a function of micelle size [12]. This function decreases initially quite steeply with $n$ and then slowly approaches its asymptotic value. This can be seen in Fig. 3, where we plotted the free energy per surfactant molecule as a function of micelle size for a single micelle at a chemical potential difference $\Delta \mu=0$. Note that the free energy goes through a minimum before it levels off. As explained in Ref. [12], the precise way in which the asymptote is approached determines if a micellar solution has a broad or narrow size distribution and even whether micelles will form, or aggregates of infinite extent in one or more dimensions (cylinders, bilayers). In the present case, it is not meaningful to discuss the asymptotic behavior, because micelles containing more than 70 surfactants are found to be mechanically unstable. In principle, the size dependence of the free energy could be analyzed in terms of macroscopic concepts (surface and curvature free energy). However, as the present simulations are limited to one type of surfactant and one state point of the solvent, such an analysis would be of limited interest.

We can use the free energy curve of Fig. 2 to recalculate the ideal size distribution. The distributions for different chemical potential difference are shown in Fig. 4. At low chemical potential difference $\Delta \mu<4.5$ there will only be clusters of a few surfactant molecules in the solution whereas at higher $\Delta \mu$ the solution is dominated by micelles 


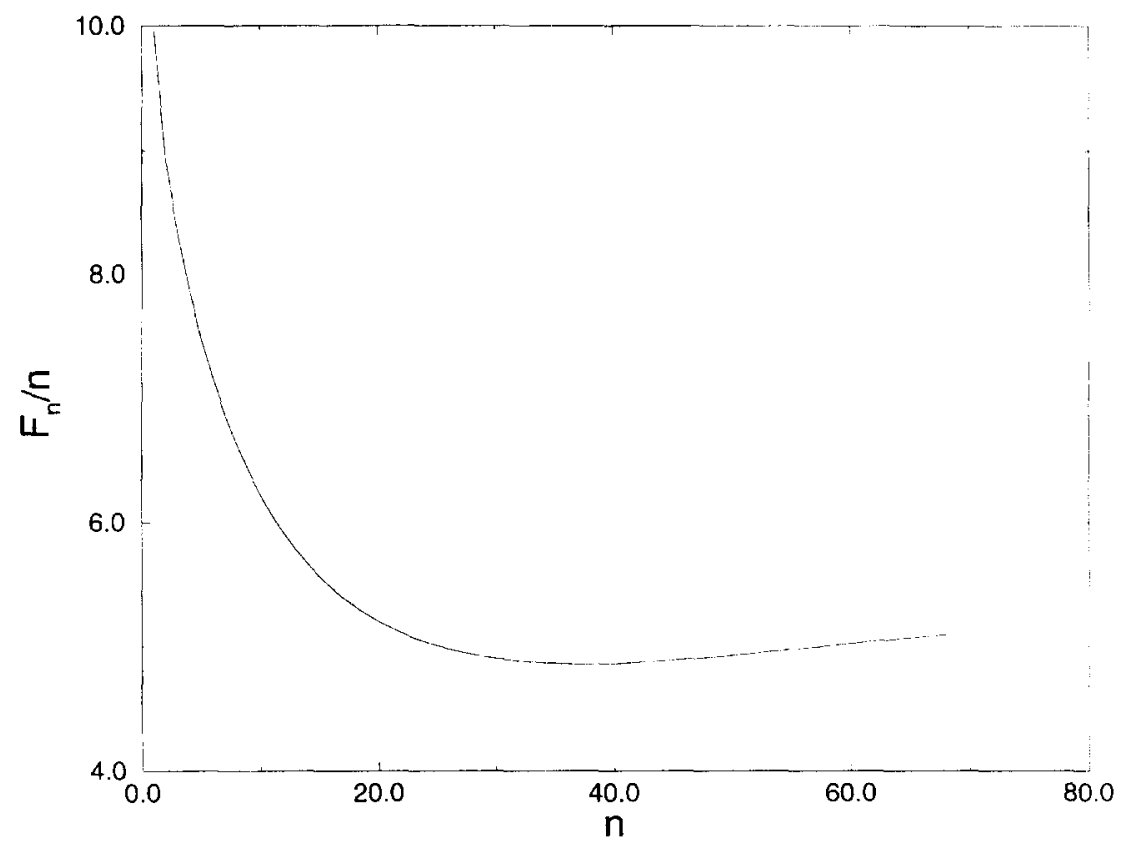

Fig. 3. Free energy per surfactant molecule of a single micelle as a function of the size (number of surfactant molecules in the micelle) for a chemical potential difference of $A \mu=0$ and at $P=8$. Note that the curve goes through a minimum.

with a size larger than 30 . Increasing the chemical potential difference results in a shift of the average micellar size. The most likely size is determined by the chemical potential of the surfactants. However, in practice, the micelle sizes are restricted to a narrow window because the total amount of surfactant increases quite rapidly with chemical potential, once the critical micelle concentration is exceeded. Hence, dilute micellar solutions can only exist for a narrow range of chemical potentials. Only a small increase in the surfactant chemical potential beyond the CMC-point, results in a situation where we can no longer ignore inter-micellar interactions. As we shall show in the next section, the effect of inter-micellar interactions is only to narrow the range of observable micelle sizes even more.

Knowledge of the micelle free energy allows us to locate the critical micelle concentration $(\mathrm{CMC})$. The definition of the $\mathrm{CMC}$ is slightly arbitrary. We define it as the concentration where half of the dissolved surfactants is in micellar clusters and the other half still exists as monomeric surfactants. We calculate the chemical potential difference at the $\mathrm{CMC}$, by equating the weighted sum of the number of surfactant molecules in a micelle of size $i$ to the number of free surfactant molecules.

$$
\sum_{i=2}^{70} i P(i, \Delta \mu)=P(1, \Delta \mu) .
$$




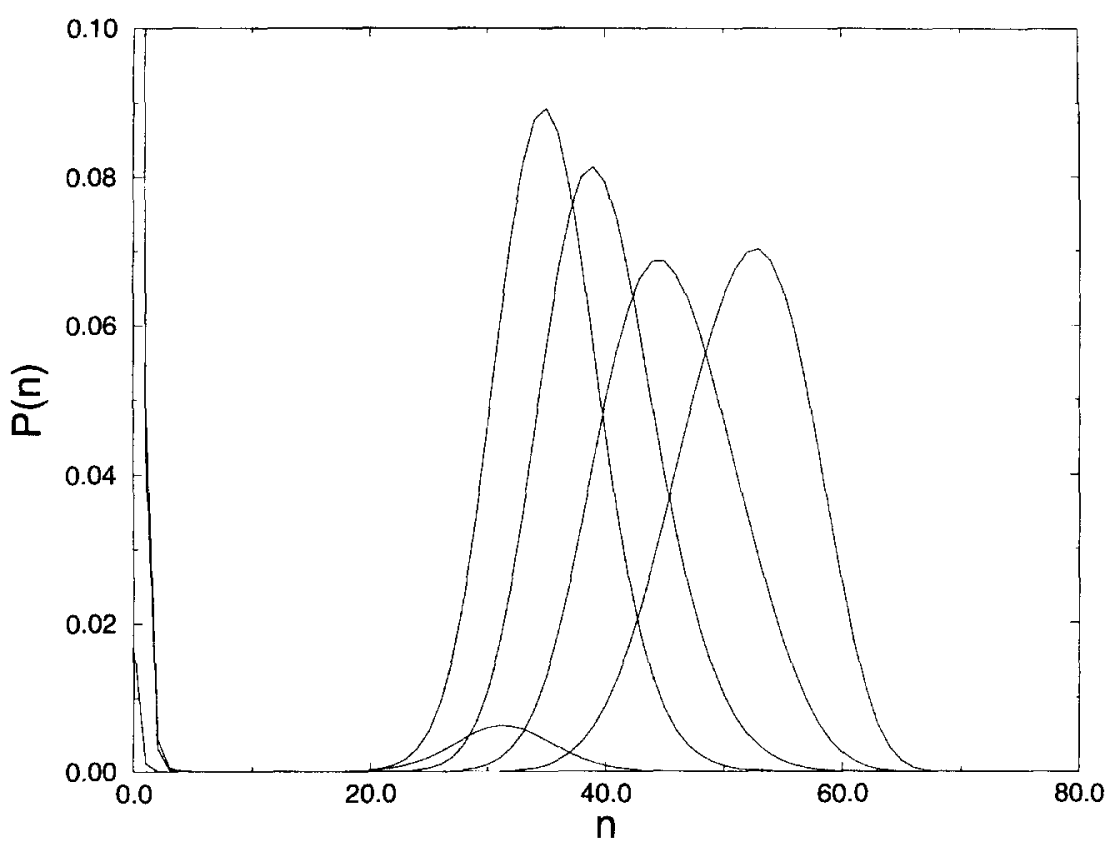

Fig. 4. Size distribution of an ideal solution of micelles. From left to right the curves correspond to a chemical potential difference of $\Delta \mu=4.2,4.4,4.6,4.8,5.0,5.2$ and 5.4. The average size of the micelles in the solution clearly increases with the chemical potential difference. Below the critical micelle concentration (corresponding to $\Delta \mu=4.50$ ), the size distribution peaks at $n=1$. However, for $\Delta \mu=4.6$, a second peak around $n=30$ is already visible. The increase in surfactant concentration with $\Delta \mu$ is very rapid: the surfactant concentration increases by a factor $10^{11}$ when $\Delta \mu$ is changed from 4.6 to 5.2. In Section 4 , we show that inter-micellar interactions change the size distribution for $\Delta \mu \geqslant 5.2$.

Solving Eq. (3) for $\Delta \mu$, we find $(\Delta \mu)_{\mathrm{CMC}}=4.50$. To express this in terms of surfactant concentrations we need to know the absolute excess chemical potential of the surfactant molecules. The most direct way to measure this would be by using a version of Widom's particle insertion scheme [13] in which one computes the probability of accepting a trial move in which a solvent molecule is transformed into a surfactant. However, the acceptance of such trial moves is very small. We therefore use an incremental growth scheme (which is more like thermodynamic integration) - we start with the monomer, and grow the surfactant tail incrementally. Every step yields a partial (incremental) chemical potential difference. In this way, we estimated the excess chemical potential difference of a surfactant molecule with respect to a solvent particle to be $\Delta \mu_{\mathrm{ex}} \approx 32$. The (ideal) number density of the surfactant $\rho_{\text {surf }}$ can be written as

$$
\log \left(\frac{\rho_{\text {surf }}}{\rho_{\mathrm{HS}}}\right)=\Delta \mu-\Delta \mu_{\mathrm{ex}} \text {. }
$$

The density of the solvent at the imposed pressure of $P=8$ is $\rho_{\mathrm{HS}}=0.66$. Substituting this density and the values for the chemical potential difference at the $\mathrm{CMC}$ and the excess difference in Eq. (4) results in a surfactant concentration at the CMC of 
$\rho_{\text {surf }}=7.5 \times 10^{-13}$ (in units $D^{-3}$ ). With present day computers, such an extremely low concentration cannot be studied in a canonical simulation.

\section{Finite density simulations}

In the previous section we showed how to obtain the size distribution of ideal solutions of micelles. However, the inter-micellar interactions become more important at higher surfactant concentration and will influence the size distribution in the system. In this section we study the effects of higher concentration on the size distributions. In principle, we could study inter-micellar interactions by performing semi-grand simulations on a large system and measure the size distribution from the simulations. However, this would require systems containing hundreds of micelles, which corresponds to tens of thousands of surfactants and even more solvent molecules. Our approach is therefore to use the information obtained in the simulation of dilute micellar solutions as input for a coarse-grained description of the dense micellar system. To this end we approximate the micelles by hard spheres of different radii and use these in a grand-canonical simulation. In doing so, we make the following assumptions:

- The micelles are effectively spherical. Snapshots of individual micelles at low concentration (see Fig. 1) show that this is a reasonable first-order approximation.

- The inter-micellar interaction is hard-sphere like. This ignores the potential of mean force due to the solvent and the effect of the deformability of micelles. There is considerable experimental evidence that, at least some, (uncharged) micelles behave as hard spheres up to quite high densities [14] - at higher densities they may still retain their identity, but the deformability becomes important [15].

For the effective hard-sphere radius of the micelles we take the average distance of the center of the head-group to the center-of-mass of the micelle, plus the radius of the head-group. We stress that all the above assumptions can easily be improved upon - we could have taken shape-polydispersity into account, and we could have measured the potential of mean force. But our primary aim is to show how we can systematically go from a molecular description of ideal micelles to a coarse-grained description of dense suspensions. If, in such a simulation, we impose a particular excess chemical potential, while ignoring the inter-micellar interactions, then we recover the size distributions of Fig. 4. If we take the inter-micellar interactions into account then we still recover the ideal size distributions at low surfactant density. In fact, it is no problem to perform a grand canonical simulation at the $\mathrm{CMC}$ where the total surfactant concentration is of order $10^{-13}$. It only requires a very large simulation box (of order $10^{15} D^{3}$ ). Such a large simulation box (in fact, if we assume that $D=1 \mathrm{~nm}$, it would correspond to a system that would even be visible with the naked eye) is computationally no problem, because the number of micelles is still low (hundreds), but clearly a "brute-force" simulation that included the solvent, would be utterly infeasible.

In order to be able to perform such semi-grand hard-sphere micelle simulations we measured the radius of the micelles as a function of the number of surfactants in 


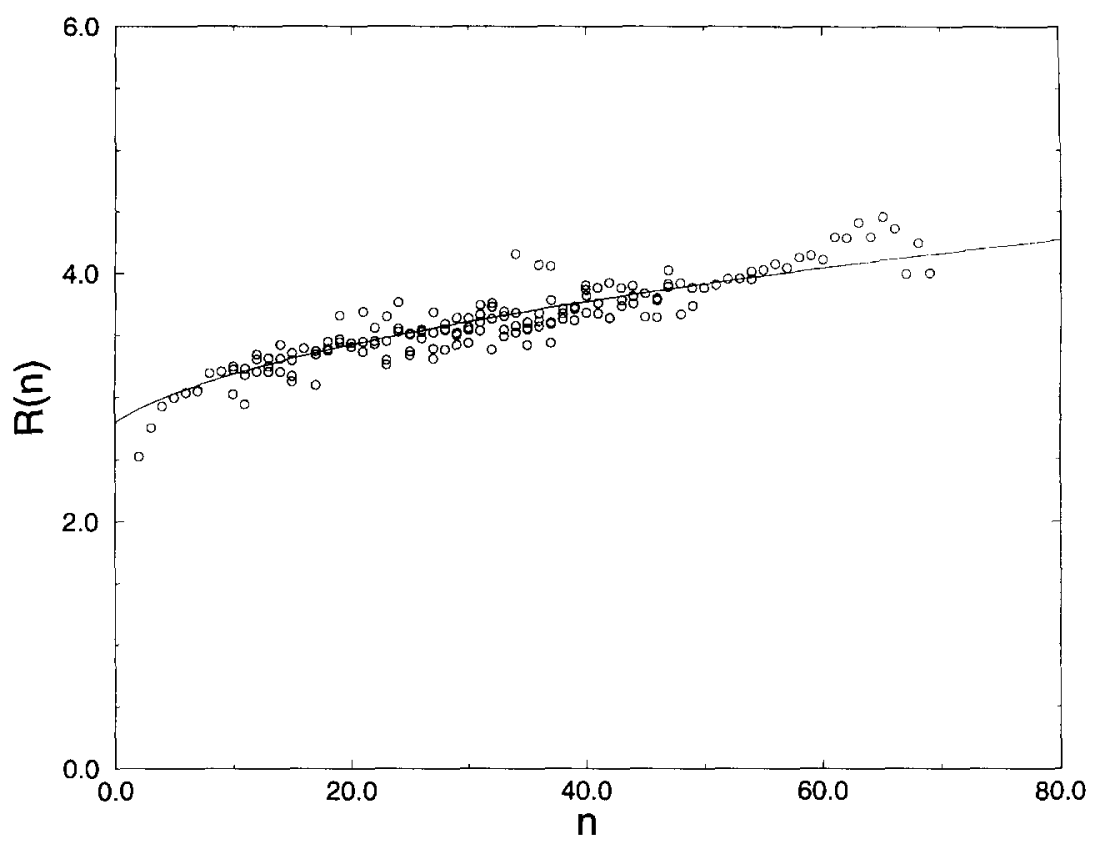

Fig. 5. Average radius of a single micelle as a function of size as obtained from the semi-grand simulations. Every open circle denotes an average over configurations of micelles containing $n$ surfactants at a particular $\Delta \mu$. The solid curve is a least squares fit to a function of the form $f(n)=a+b \sqrt{n}$. This fit was used in the polydisperse hard sphere grand canonical simulations.

the micelle from the single micelle simulations described in the previous section. In Fig. 5, the measured radius is plotted. A striking feature of this figure is that the radius changes only slowly with size. This is probably due to the fact that we use a rigid surfactant tail. Even in small clusters the head groups are forced to be separated by at least a tail length. When more surfactant molecules are added, the shell of head groups will fill up, without appreciably increasing the radius of the cluster significantly. However, at high packing fraction of head-groups in the shell, there is a tendency of the micelle to increase its surface. If the micelle would remain spherical, this would result in an increase in volume. The simulations suggest that this expansion becomes appreciable around $n=64$. However, large spherical micelles are apparently not stable and for $n \approx 70$, the micelle breaks up into two separate clusters. We stress, however, that the details of this scenario depend on the model used.

Fig. 6 shows the size distributions that we measured in the grand-canonical simulations, together with the corresponding ideal distributions. At high concentrations, the influence of inter-micellar interactions is clearly visible. The interactions between the micelles, shift the size distributions to lower average sizes.

In fact, the size distributions of the interacting system closely resemble those of the non-interacting system at a lower chemical potential. We can therefore fit the size distribution of interacting micelles to that of ideal micelles at a different chemical 


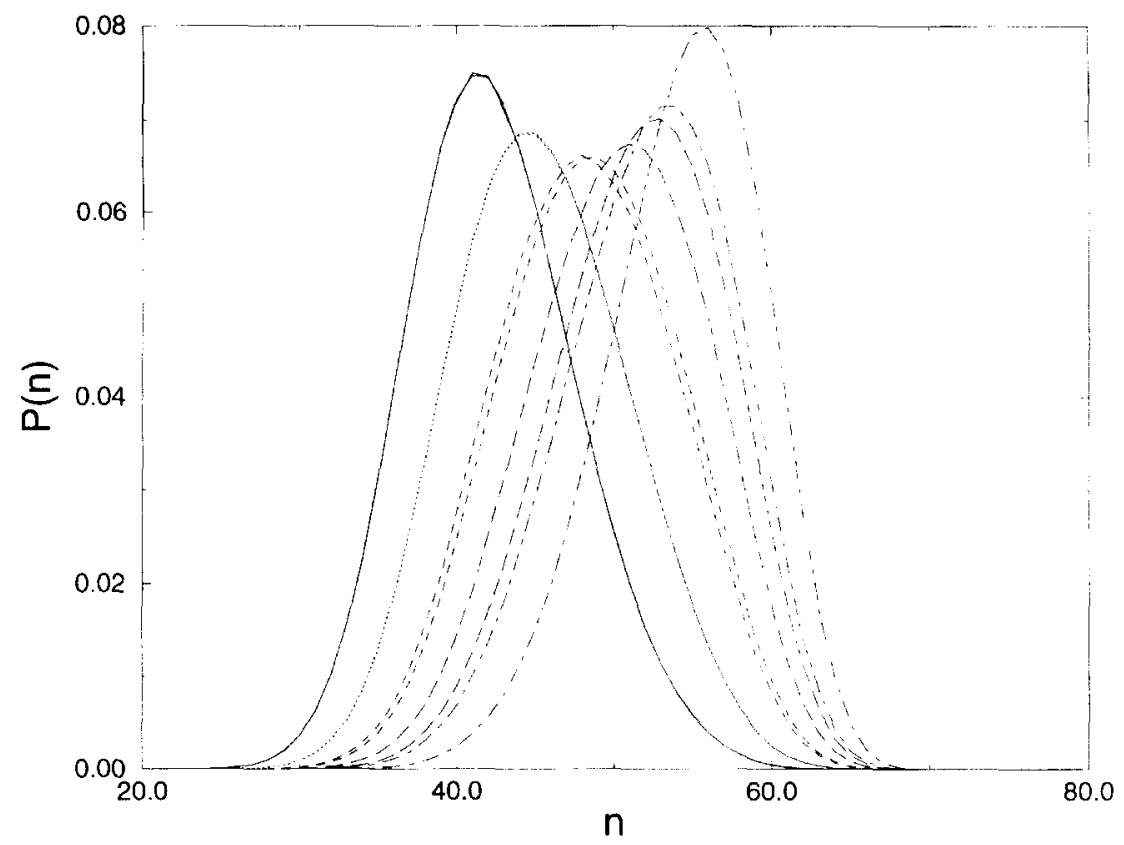

Fig. 6. Influence of inter-micellar interaction on the size distribution in a micellar solution. For comparison the ideal size distribution is also plotted. The size distribution of the interacting micellar solution is always shifted to the left. The different curves correspond to a chemical potential difference of $\Delta \mu=5.1$ ( solid), 5.2 (dotted), 5.3 (dashed), 5.4 (long dashed) and 5.45 (dot-dashed). In every case, the effect of interactions is to shift the maximum of the distribution to smaller particle sizes.

potential. We refer to the chemical potential that yields the best fit as the "effective ideal" chemical potential. In Fig. 7 we have plotted the difference between the real surfactant chemical potential and the effective ideal chemical potential, as a function of the total volume fraction occupied by the micelles.

This behavior is to be expected as long as the excess chemical potential depends linearly on the number of particles in a cluster. We can expand the excess chemical potential in a Taylor series around the average cluster size. For moderate polydispersities, the linear term in this Taylor series will dominate.

In order to estimate the shift in the effective chemical potential (Fig. 7), we need to know how the excess free energy of a (polydisperse) hard-sphere fluid depends on the number of surfactants in the spheres. It is straightforward to estimate how the excess free energy of hard spheres varies with an infinitesimal change in the radius. We combine this with the observed relation between the micellar radius and the number of surfactants (Fig. 5). If we ignore polydispersity, the relation between $F_{\mathrm{ex}}$ and $n$ then becomes:

$$
\frac{\partial F_{\mathrm{ex}}}{\partial n}=\left(\frac{\partial F_{\mathrm{ex}}}{\partial \phi}\right)\left(\frac{\partial \phi}{\partial r}\right)\left(\frac{\partial r}{\partial n}\right)=3(Z(\phi)-1) \frac{d \ln r}{d n},
$$




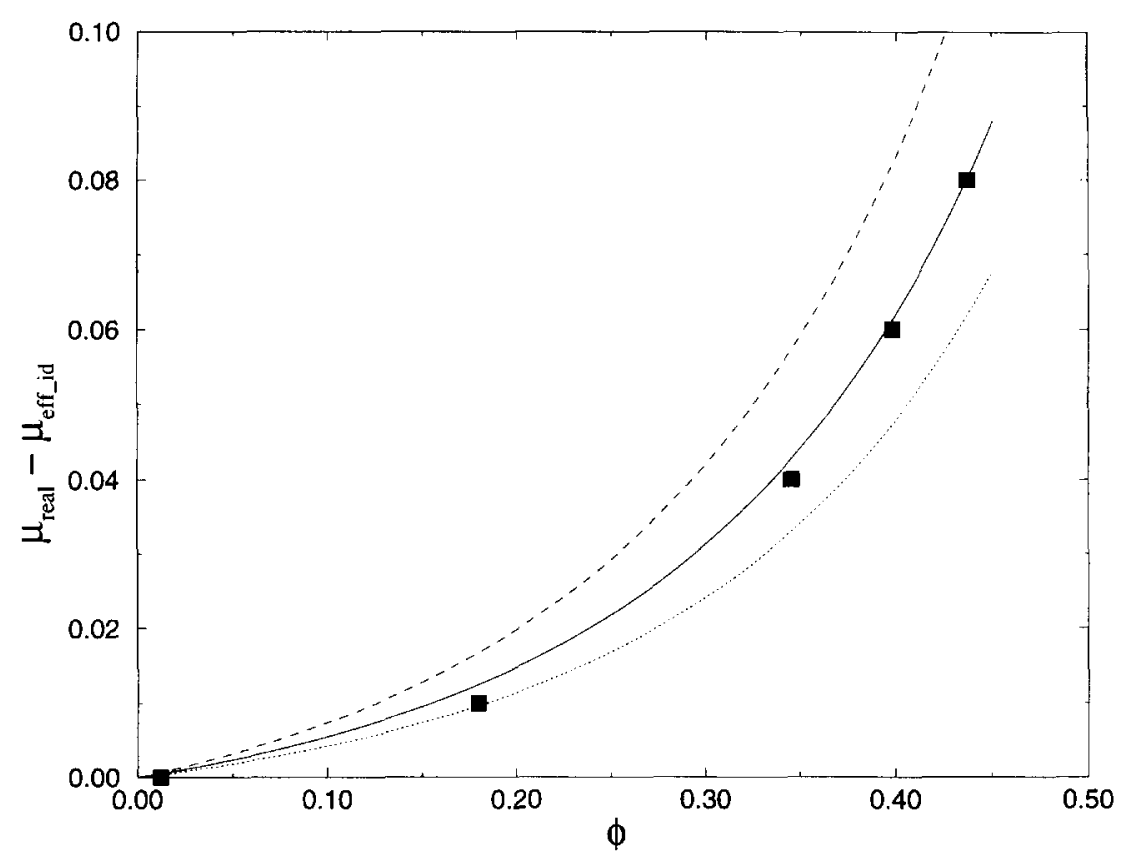

Fig. 7. The size distribution of interacting micelles can be described by the size distribution of ideal (non-interacting) micelles at a slightly lower value of the chemical potential. The figure shows this difference $\mu_{\text {real }}-\mu_{\text {eff }}$, as a function of the volume fraction of micelles. The filled squares denote the simulation results. The drawn curve was obtained using the "mean-field" prediction (Eq. (6)) described in the text. This curve was obtained using the average value of $d \ln r / d n$ for $30 \leqslant n \leqslant 70$. To show the sensitivity of the results to $d \ln r / d n$, we have also plotted the predictions for a value of $d \ln r / d n$ that is $10 \%$ too high (dashed curve) and 10\% too low (dotted curve).

where $Z_{\mathrm{CS}}$ is the compressibility factor $P /(\rho k T)$. We assume that the equation of state of the suspension is adequately described by the Carnahan-Starling equation of state for hard spheres (which is reasonable if we can ignore polydispersity). The derivative of $F_{\mathrm{ex}}$ with respect to $n$ is then given by:

$$
\frac{\partial F_{\mathrm{ex}}}{\partial n}=3\left(\frac{1+\phi+\phi^{2}-\phi^{3}}{[1-\phi]^{3}}-1\right)\left(\frac{d \ln r}{d n}\right),
$$

where $Z_{\mathrm{CS}}$ is the Carnahan-Starling compressibility factor. In Fig. 7 we show the resulting estimate of the shift in effective chemical potential. In view of the extreme simplicity of this theory, the agreement is gratifying. Its success indicates that the influence of inter-micellar interactions on the particle-size distribution can, to a first approximation, be thought of as a mean-field effect. In fact, the present approach can easily be extended to the more general situation that the effective inter-micellar potential is of the form $u(r)=\varepsilon f(r / \sigma)$. For hard micelles, it is possible to improve on Eq. (6) by employing the Mansoori equation of state for polydisperse hard spheres [16]. We have done this and find that the agreement between a mean-field theory based on the Mansoori equation-of-state and the simulation results is essentially quantitative. 
However, the predictive power of this more sophisticated theory is limited because it requires the computed micellar size-distribution as input.

\section{Conclusions}

This paper illustrates that semi-grand ensemble simulations are very convenient for an accurate determination of the free energy of isolated micelles. Use of the semi-grand ensemble results in a much faster equilibration of the micelle distribution than can be achieved in canonical simulations. The measured free energy as a function of micelle size shows a distinct barrier for the formation and a minimum at the average size. This average size of the micelles depends on the surfactant concentration (chemical potential difference of the reservoir). Once the micelle free-energy function is known, it is straightforward to locate the CMC.

By approximating the micelle solution as a polydisperse hard sphere system, the effect of inter-micellar interactions on the size distributions could be efficiently studied by means of a grand-canonical simulation. At high surfactant concentrations, intermicellar interactions have a pronounced effect on the size distribution. However, for the present system, even at fairly high packing fractions, the particle size distribution is well reproduced by a distribution of ideal (non-interacting) micelles at a lower chemical potential.

We note that micelles become unstable for $n \geqslant 70$. This is interesting, because it suggests that there are two possible scenarios for the kinetics of micelle formation. The first is that micelles simply form by aggregation of monomers. But the second scenario, that becomes more likely at higher surfactant concentrations, is that an existing micelle grows beyond the critical size and breaks up to form two micelles. The ratio of the heights of the free energy barriers for these two processes depends on the surfactant chemical potential.

The techniques described in this paper can be extended to describe micelles with pair-interactions that are not hard-sphere like. This would make it possible to perform "coarse-grained" simulations of dense suspensions of realistic models for micelles.

\section{Acknowledgements}

The work of the FOM Institute is part of the research program of FOM and is made possible by financial support from the Netherlands Organization for Scientific Research (NWO). We thank Richard Sear for a critical reading of the manuscript.

\section{References}

[1] W.W. Wood, F.R. Parker, J. Chem. Phys. 27 (1957) 720.

[2] B. Widom, J.S. Rowlinson, J. Chem. Phys. 52 (1970) 1670. 
[3] B. Widom, J. Phys. Chem. 88 (1984) 6508.

[4] B. Widom, J. Chem. Phys. 84 (1986) 6943

[5] D.A. Kofke, E.D. Glandt, Mol. Phys. 64 (1988) 1105.

[6] B. Smit, P.A.J. Hilbers, K. Esselink, L.A.M. Rupert, N.M. van Os, A.G. Schlijper, Nature 348 (1990) 624; B. Smit, P.A.J. Hilbers, K. Esselink, L.A.M. Rupert, N.M. van Os, J. Phys. Chem. 95 (1991) 6361.

[7] J.C. Desplat, C.M. Care, Mol. Phys. 87 (1996) 441.

[8] D.R. Rector, F. van Swol, J.R. Henderson, Mol. Phys. 82 (1994) 1009.

[9] A.T. Benardes, V.B. Henriques, P.M. Bisch, J. Chem. Phys. 101 (1994) 645.

[10] D. Frenkel, B. Smit, Understanding Molecular Simulation, Academic Press, Boston, 1996.

[11] G.M. Torrie, J.P. Valleau, J. Comp. Phys. 23 (1977) 187.

[12] J.N. Israelachvili, D.J. Mitchell, B.W. Ninham, J. Chem. Soc. Faraday Trans II 72 (1976) 1525.

[13] B. Widom, J. Chem. Phys. 39 (1963) 2808.

[14] M. Giustini, G. Palazzo, G. Colafemmina, M. Della Monica, M. Giomini, A. Ceglie, J. Phys. Chem. 100 (1996) 3190.

[15] T.G. Mason, J. Bibette, D.A. Weitz, Phys. Rev. Lett. 75 (1995) 2051; M.D. LaCasse, G.S. Grest, D. Levine, T.G. Mason, D.A. Weitz, Phys. Rev. Lett. 76 (1996) 3448.

[16] G.A. Mansoori, N.F. Carnahan, K.E. Starling, T.W. Leland, J. Chem. Phys. 54 (1971) 1523. 